\title{
Appendix 2 Interview Questions
}

\section{Key}

Text in italics indicates probes/questions added throughout data collection as part of an iterative process.

\section{Verbal Boundaries}

(Biographies were obtained prior to interview)

1. Please provide a brief overview of your experience of using rap in music therapy including settings, context (group or individual work) and client groups?

- $\quad$ how familiar and comfortable are you with the Rap genre?

- did this develop over time?

- Which type/styles of Rap do clients bring (in terms of verbal content)?

- how much do you allow people to use words to express feelings, even if offensive?

- more concerning/bigger things to think about?

2. Were there instances when you needed to consider boundaries regarding lyrical content? If so, can you describe these instances and explain how you dealt with them?

- what type of lyrical content required you to consider boundaries (e.g. expletives, violence)?

- why?

- setting and MDT/team?

- culture of clients?

3. What's your stance on the use of offensive language in music therapy and how do you manage it?

- general verbal content

- rap content

- if person has used rap in various settings/with various client groups how does this differ/what are similarities?

- how much do you allow people to use words to express feelings, even if offensive?

- more concerning/bigger things to think about?

- Modelling?

- Metaphoric and not literal? 
4. What are your views on rap that could be described as gangsta rap (elevates violence, crime, drug use etc. and is particularly extreme in it's use of offensive language) within music therapy and how would you manage its use in a session?

- mimicking offensive lyrics

- contraindicative

- cathartic form of expression

5. In your experience, how does the management of boundaries affect the therapeutic relationship?

- would prohibiting offensive language hinder the therapeutic relationship/get in way?

- have clients set their own boundaries?

- how does the clients use of offensive language impact on you and your counter transference reaction?

- OR do your feelings (scared/abused) inform you of what person trying to communicate?

- Experience - has this changed over time in relation to counter transference?

- False persona/needing to protect themselves

6. What effect does management of boundaries have on the music that's made?

- preserving craft/style

- distracting from making music?

\section{Contrainidication}

7. Can you think of any examples of when the use of rap may be inappropriate or contraindicative and if this was your own work, how did you manage this?

- 'acting out' - positive or negative?

- channelled/sublimated - positive

- paralleling offensive behaviours - negative

- negative role models?

- supervision?

8. What impact can Rap have on group members and group processes?

- contraindicative for individuals who do not enjoy listening to Rap

- MT suggested use of rap?

- Client brought in?

- Group decided on rap? 
9. Do the stylistic and cultural connotations of the rap genre have an influence on management of verbal boundaries and if so, how?

- $\quad$ keepin' it real'

- fantasy vs. reality

- where can it help and where can it hinder?

- society consistently disparages rap as violent and negative

- Many clients who bring gangsta rap have been exposed to the most extreme life circumstances 